\title{
Revisiting Kawasaki dynamics in one dimension
}

\author{
M. D. Grynberg \\ Departamento de Física, Universidad Nacional de La Plata, 1900 La Plata, Argentina
}

(Received 22 September 2010; published 16 November 2010)

\begin{abstract}
Critical exponents of the Kawasaki dynamics in the Ising chain are re-examined numerically through the spectrum gap of evolution operators constructed both in spin and domain-wall representations. At lowtemperature regimes the latter provides a rapid finite-size convergence to these exponents, which tend to $z$ $\simeq 3.11$ for instant quenches under ferromagnetic couplings, while approaching to $z \simeq 2$ in the antiferro case. The spin representation complements the evaluation of dynamic exponents at higher temperature scales, where the kinetics still remains slow.
\end{abstract}

DOI: 10.1103/PhysRevE.82.051121

PACS number(s): 02.50.-r, 05.50.+q, 75.78.Fg, 64.60.Ht

\section{INTRODUCTION}

Kinetic Ising models have long been helpful in understanding general questions of nonequilibrium statistical physics, as well as for elucidating particular issues of experimental relevance [1]. There is by now a vast body of research studying these models under Glauber and Kawasaki dynamics [2,3], the so-called models $A$ and $B$, respectively, in the terminology of Hohenberg and Halperin [4]. The first type of dynamic considers single spin flip processes for describing relaxation toward equilibrium in a variety of magnetic materials, while the second case consists of spin exchanges specially aimed to study stochastic processes under conservation of total magnetization. This constraint makes relaxation slower and is instrumental in studying phase separation, domain growth, and freezing observed after rapid cooling in several systems, such as binary fluids and alloys [1]. All these phenomena are characterized by a long relaxation time $\tau$ which near critical points diverges as $\tau \propto \xi^{z}$, where $\xi$ denotes the spatial correlation length (proportional to the typical system size) and $z$ is the dynamic exponent of the universality class to which the dynamic belongs.

When it comes to one dimension (1D), these dynamics are still largely amenable to experimental probe [5] apart from being interesting in their own right [6], and as is well known, in the Glauber case allow for an exact treatment [2,5]. In contrast to that latter aspect, the equations of motion for the Kawasaki dynamics conform an infinite hierarchy which do not admit analytic solution, so the problem can only be approached from approximations [5]. Even though there is no finite critical temperature through which to quench, using random-walk arguments to derive an approximate kinetic equation for the kink density under ferromagnetic $(\mathrm{F})$ interactions [7], interestingly, the dynamic exponent has been put forward to be the same as observed in higher dimensions, namely, $z=3$, after a deep quench to a low temperature (LT). Although for $d \geq 2$ such exponent was also accounted for by renormalization-group arguments $[1,8]$ as well as by surface dynamical considerations [9], other studies in 1D related systems [10] suggest instead a value of $z=5$ [11], in agreement with linear-response schemes [12].

In this work we re-examine this exponent by constructing and diagonalizing numerically the kink evolution operator associated to the master equation of the Kawasaki dynamics in finite chains. Following the physical picture given in [7], at late evolution stages and LT regimes such operator would essentially describe a highly diluted system as the kink or domain-wall density is basically a measure of the average inverse domain size. Consequently, in evaluating dynamic exponents from finite samples it might be expected that size effects will pose no severe limitations for the kink representation. In fact, as we shall see, the kink density will get fairly small even for the low lying levels of the evolution operator, that is what ultimately matters at large times. On the other hand there is also the question about estimating dynamic exponents at finite temperatures for which the Kawasaki kinetics still remains critical, as opposed to the exponential decay of the Glauber dynamics in 1D. Although in this situation dynamic exponents are no longer related to domain growth - these are cut off by a finite correlation length-they still provide the fundamental relation between the typical system size and its relaxation time. We shall also address this issue by diagonalizing the evolution operator of the original spins. Insofar temperature is not too low, these latter become weakly correlated and finite-size effects will not be paramount. A posteriori, our results will lend further support to this view.

Therefore these dual representations-kinks and spinsprovide a means for probing the robustness of dynamic exponents throughout different regimes. As a result, it will turn out that for $\mathrm{F}$ couplings $z$ varies continuously from $\simeq 3.11$ to $\simeq 2$ at low and high-temperature (HT) scales, respectively. Such nonuniversality should come as no surprise since in the HT limit the Kawasaki dynamics simply reduces to a diffusive disordering kinetics. However under antiferromagnetic (AF) exchanges these exponents are rather robust and, in line with previous findings [10], their values will remain diffusive even at LT regimes, just as the $z$ of the Glauber universality class. We shall return to this question later on within a context of antikink operators.

The layout of this work is organized as follows. In Sec. II we recast the $1 \mathrm{D}$-Kawasaki dynamics in terms of a quantum spin analogy that readily lends itself to evaluate spectrum gaps of evolution operators, i.e., dynamic exponents, both in the spin and domain-wall representations. Due to detailed balance, either of these descriptions can be brought to a symmetric representation by means of simple nonunitary spin rotations. This simplifies considerably the subsequent numerical analysis of Sec. III in which spectrum gaps are ob- 
tained via standard recursive techniques [13] in various situations. For now let us remark that already modest chain lengths are able to yield clear finite-size trends both at LT and HT scales. Finally, Sec. IV contains a summarizing discussion along with some remarks on extensions of this work.

\section{SPIN AND KINK REPRESENTATIONS}

As is well known, the Ising model has no intrinsic dynamics because all spin operators involved in its Hamiltonian commute with one another. Therefore, an ad hoc dynamics must be prescribed by coupling the system to a heath bath at temperature $T$ so as to induce energy changes in the model. This is described by a master equation [14] for which we introduce briefly here some preliminary considerations.

Basically, the dynamics is associated to a gain-loss equation, constructed generically as

$$
\partial_{t} P(s, t)=\sum_{s^{\prime}}\left[W\left(s^{\prime} \rightarrow s\right) P\left(s^{\prime}, t\right)-W\left(s \rightarrow s^{\prime}\right) P(s, t)\right],
$$

which governs the evolution of the probability $P(s, t)$ that the system will be at state $|s\rangle$ at time $t$. The elementary change steps are embodied in the transition probability rates $W(s$ $\rightarrow s^{\prime}$ ) per unit time at which configuration $|s\rangle$, evolves to $\left|s^{\prime}\right\rangle$. For our purposes, it is convenient to think of this equation as a Schrödinger evolution in an imaginary time, namely, $\partial_{t}|P(t)\rangle=-H|P(t)\rangle$, under a pseudo-Hamiltonian or evolution operator $H=H_{d}+H_{n d}$ whose diagonal and nondiagonal matrix elements are given by

$$
\begin{aligned}
& \left\langle s\left|H_{d}\right| s\right\rangle=\sum_{s^{\prime} \neq s} W\left(s \rightarrow s^{\prime}\right), \\
& \left\langle s^{\prime}\left|H_{n d}\right| s\right\rangle=-W\left(s \rightarrow s^{\prime}\right) .
\end{aligned}
$$

Formally, this enables one to derive all subsequent probability distributions $|P(t)\rangle \equiv \Sigma_{s} P(s, t)|s\rangle$, from the action of $H$ on a given initial distribution, i.e., $|P(t)\rangle=e^{-H t}|P(0)\rangle[14]$. In particular, the relaxation time of any observable with nonzero matrix element between the steady state and the first excitation mode of $H$ is singled out by the eigenvalue $\lambda_{1}$ corresponding to this latter, i.e., $\tau^{-1}=\operatorname{Re}, \lambda_{1}>0$, whereas the former merely corresponds to an eigenvalue $\lambda_{0}=0$ [14]. If the steady solution should actually coincide with the Boltzmann equilibrium distribution, the above rates must be chosen to satisfy detailed balance, that is, $W\left(s \rightarrow s^{\prime}\right)$, $e^{-\beta E(s)}=W\left(s^{\prime} \rightarrow s\right), e^{-\beta E\left(s^{\prime}\right)}, \forall s, s^{\prime}$, where $E$ stands for the respective energy configurations of the system in question and $\beta \equiv 1 / k_{B} T$.

Detailed balance itself cannot determine entirely the form of such rates, thus for the specific case of the Kawasaki dynamics hereafter considered we take up the common choice $[1,3]$

$$
W\left(s \rightarrow s^{\prime}\right)=\frac{1}{2}\left[1-\tanh \left(\frac{\beta}{2} \Delta E_{s, s^{\prime}}\right)\right],
$$

where $|s\rangle,\left|s^{\prime}\right\rangle$ are states differing at most in a pair of nearest-neighbor (NN) spins exchanged at some location

$$
\begin{gathered}
R_{i-1, i+2}(K) \\
s_{i-1} \uparrow \downarrow s_{i+2} \rightleftharpoons s_{i-1} \downarrow \uparrow s_{i+2} \\
R_{i-1, i+2}(-K)
\end{gathered}
$$

FIG. 1. Transition probability rates for neighboring spin exchanges, as defined in Eq. (5).

$i=1,2, \ldots, L$, and $\Delta E_{s, s^{\prime}} \equiv E\left(s^{\prime}\right)-E(s)$ is the change in the Ising energy $E=-J \sum_{i} s_{i} s_{i+1},\left(s_{i}= \pm 1\right)$ either with $\mathrm{F}(J>0)$ or AF $(J<0)$ interactions. Therefore depending on the spin states at locations $i-1$ and $i+2$, the rate at which spins $\left(s_{i}, s_{i+1}\right)=(s,-s)$ exchange their states results in

$$
R_{i-1, i+2}( \pm K)=\frac{1}{2} \pm \frac{\alpha_{K}}{4}\left(s_{i+2}-s_{i-1}\right),
$$

where $K=\beta J, \alpha_{K}=\tanh 2 K$, and the signs \pm denote forward and backward hoppings as depicted schematically in Fig. 1.

If we think of the spin configurations $|s\rangle$ as being already diagonal in the $z$ direction, say, then by promoting $s_{i}$ variables to Pauli matrices $\sigma_{i}^{z}$, the operational analog of Eq. (3) will read

$$
H_{n d}=-\sum_{i}\left[R_{i-1, i+2}^{z}(K) \sigma_{i+1}^{+} \sigma_{i}^{-}+R_{i-1, i+2}^{z}(-K) \sigma_{i}^{+} \sigma_{i+1}^{-}\right]
$$

where $\sigma^{+}, \sigma^{-}$are the usual spin- $\frac{1}{2}$ raising and lowering operators, while $R_{i-1, i+2}^{z}( \pm K)=\frac{1}{2} \pm \frac{\alpha_{K}}{4}\left(\sigma_{i+2}^{z}-\sigma_{i-1}^{z}\right)$, is simply the operational counterpart of Eq. (5). As for the diagonal elements of Eq. (2) needed for conservation of probability, notice that these basically count the number of hoppings in which a given configuration can evolve to different ones by exchanging NN spins at a time. This can be properly tracked down in terms of number operators $\hat{n}=\sigma^{+} \sigma^{-}$probing and weighting all NN spins, namely,

$$
H_{d}=\sum_{i}\left[R_{i-1, i+2}^{z}(K) \hat{n}_{i}\left(1-\hat{n}_{i+1}\right)+R_{i-1, i+2}^{z}(-K) \hat{n}_{i+1}\left(1-\hat{n}_{i}\right)\right]
$$

Although the correlated exchange terms of Eq. (6) leave us with a nonsymmetric evolution operator, in preparation for the numerical analysis of Sec. III we can make some progress by exploiting detailed balance. This latter warrants the existence of representations in which $H$ is symmetric and thereby diagonalizable [14]. For our purposes, it suffices to consider the diagonal nonunitary similarity transformation

$$
S=e^{-K / 2 \Sigma_{j} \sigma_{j}^{z} \sigma_{j+1}^{z}}
$$

under which the hopping terms of $H_{n d}$ transform as

$$
\sigma_{i+1}^{ \pm} \sigma_{i}^{\mp} \rightarrow \exp \left[\mp K\left(\sigma_{i+2}^{z}-\sigma_{i-1}^{z}\right)\right] \sigma_{i+1}^{ \pm} \sigma_{i}^{\mp},
$$

while leaving invariant all the above mentioned diagonal operators. Hence, after straightforward manipulations it can be readily verified that $H_{\text {spin }}=S H S^{-1}$ actually produces a self adjoint spin representation which for periodic boundary conditions (PBCs) is found to be 


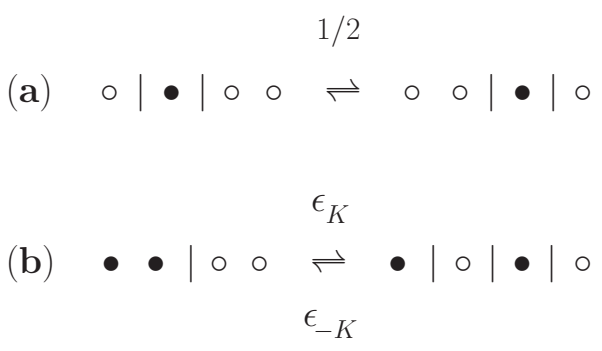

FIG. 2. Transition rates for (a) diffusion of double kinks and (b) assisted deposition-evaporation of kinks. These latter are denoted by vertical lines separating domains of opposite spin orientations.

$$
\begin{aligned}
\mathrm{H}_{\mathrm{spin}}= & -\frac{1}{8}\left(1+\gamma_{K}\right) \sum_{i}\left(1+\tanh ^{2} K \sigma_{i-1}^{z} \sigma_{i+2}^{z}\right)\left(\sigma_{i}^{x} \sigma_{i+1}^{x}+\sigma_{i}^{y} \sigma_{i+1}^{y}\right) \\
& +\frac{1}{4} \sum_{i}\left[1+\alpha_{K} \sigma_{i}^{z} \sigma_{i+2}^{z}-\left(1+\alpha_{K}\right) \sigma_{i}^{z} \sigma_{i+1}^{z}\right],
\end{aligned}
$$

where $\gamma_{K}=\operatorname{sech} 2 K$. In passing, it is worth pointing out that in the HT limit this operator coalesces into a Heisenberg ferromagnet, i.e., $H_{\text {spin }} \rightarrow-\frac{1}{4} \sum_{i}\left(\vec{\sigma}_{i} \cdot \vec{\sigma}_{i+1}-1\right)$, with a spectrum gap behaving like $\propto 1 / L^{2}$, as it should for a disordering diffusive kinetics. On the other hand, in such regime the equilibrium correlation length becomes of the order of the lattice spacing, thus the numerical diagonalization of $H_{\text {spin }}$ in finite chains can be a reasonable starting point to evaluate dynamic exponents as temperature is quenched slightly (though instantly to a constant value, because transition rates have been taken time independent throughout).

When it comes to LT regimes however, Eq. (10) is not of much practical use, since the system approaches a state consisting of long magnetic domains whose typical sizes are of the order of the correlation length $\xi \sim e^{2|K|}$ (nonetheless, for $J>0$ see the upper bounds to $z$ and their extrapolations provided in Sec. III A). In this more interesting scenario the dynamics is strongly affected by whether the coupling exchanges are $\mathrm{F}$ or $\mathrm{AF}$, thus it is convenient at this point to separate the discussion accordingly.

\section{A. Kink representation: $\boldsymbol{J}>\mathbf{0}$}

As was mentioned in Ref. [7], the dynamics of this situation is basically mediated by single spins detaching from a domain wall (a kink), and then performing a random walk. Some of these spins will return to their original domains, while others will reach the next ones. On average, as a result of many of these processes the domains themselves will perform a random walk until they meet and merge into larger domains. Besides, single meandering spins may eventually nucleate and trigger the growth of a new domain. The key issue in this description is that for large times very few kinks will survive at LT scales. In particular, in equilibrium these are totally uncorrelated and with an average density $\sim 1 / \xi$, whereas that of the spins mediating the whole process is of order $1 / \xi^{2}$ as they are made up of two consecutive kinks [see Fig. 2(a) below]. Thus, for large times it makes sense to attempt a nonequilibrium description in terms of kinks rather than spins. Presumably (as confirmed later on in Sec. III), the first excitation mode of the kink evolution operator will also correspond to a diluted state, so size effects in a numerical estimation of $z$ will not be as severe as in the spin description.

To recover the equilibrium behavior of the Ising model from now on we consider the case of zero magnetization, so this dual representation corresponds to the two-to-one mapping outlined schematically in Fig. 2. If we imagine these kinks as hard core particles $A$, then the Kawasaki dynamics involve two basic processes (i) dimer diffusion $A+A$ $+\varnothing \rightleftharpoons \varnothing+A+A$, and (ii) "assisted" deposition-evaporation $\varnothing+A+\varnothing \rightleftharpoons A+A+A$, as schematized, respectively, in Figs. 2(a) and 2(b). The first situation represents the meandering spins referred to above and involves no energy changes (rates 1/2). Notice that these dimers (double kinks) do not preserve their identity as they may eventually dissociate when contacting a domain wall (single kink). The second process corresponds to a spin detachment (attachment) from a domain wall, and its rates $\epsilon_{K}\left(\epsilon_{-K}\right)$ parallel those of Eq. (5) when $s_{i-1}=-s_{i+2}$, thus

$$
\epsilon_{ \pm K}=\frac{1}{2}(1 \mp \tanh 2 K) .
$$

Therefore, following the reasoning steps discussed for the spin representation and reinterpreting kinks as $\frac{1}{2}$-spinors in a dual chain, clearly the nondiagonal terms $H_{n d}^{a}+H_{n d}^{b}$ of the evolution operator associated to the (a) and (b) processes of Fig. 2 can be constructed as

$$
\begin{gathered}
H_{n d}^{a}=-\frac{1}{2} \sum_{i} \hat{n}_{i}\left(\sigma_{i+1}^{+} \sigma_{i-1}^{-}+\text {H.c. }\right), \\
H_{n d}^{b}=-\sum_{i} \hat{n}_{i}\left(\epsilon_{K} \sigma_{i-1}^{+} \sigma_{i+1}^{+}+\epsilon_{-K} \sigma_{i-1}^{-} \sigma_{i+1}^{-}\right),
\end{gathered}
$$

with number operators acting here as projectors that rule out vacancy mediated processes. To recast the nondiagonal operator into a symmetric representation, we recur once more to detailed balance and rotate all $\sigma^{+} \mathrm{s}$ around the $z$ direction using a common pure imaginary angle $\varphi=i K$. This is carried out by the nonunitary similarity transformation

$$
\mathrm{S}=e^{K / 2 \Sigma_{j} \sigma_{j}^{z}}
$$

for which it is straightforward to show that

$$
\sigma_{i-1}^{ \pm} \sigma_{i+1}^{ \pm} \rightarrow e^{ \pm 2 K} \sigma_{i-1}^{ \pm} \sigma_{i+1}^{ \pm},
$$

thus producing the symmetrization of $H_{n d}^{b}$ while keeping all terms of $H_{n d}^{a}$ (already Hermitian) unaltered.

As before, preservation of probability is taken into account by a diagonal operator $H_{d}^{a}+H_{d}^{b}$ balancing both of the above (mutually exclusive) events, in turn probed, respectively, by

$$
H_{d}^{a}=\frac{1}{2} \sum_{i}\left[\hat{n}_{i-1} \hat{n}_{i}\left(1-\hat{n}_{i+1}\right)+\left(1-\hat{n}_{i-1}\right) \hat{n}_{i} \hat{n}_{i+1}\right],
$$




$$
H_{d}^{b}=\sum_{i}\left[\epsilon_{K}\left(1-\hat{n}_{i-1}\right) \hat{n}_{i}\left(1-\hat{n}_{i+1}\right)+\epsilon_{-K} \hat{n}_{i-1} \hat{n}_{i} \hat{n}_{i+1}\right]
$$

Since the imaginary rotation Eq. (14) is also diagonal, it has no effect on these latter operators. Thus, collecting all terms and using PBC throughout, after some algebraic steps we finally obtain a self adjoint representation for the kink evolution operator, namely,

$$
\begin{aligned}
H_{\mathrm{kink}}= & -\frac{1}{8} \sum_{i}\left(1+\sigma_{i}^{z}\right)\left[\left(1+\gamma_{K}\right) \sigma_{i-1}^{x} \sigma_{i+1}^{x}+\left(1-\gamma_{K}\right) \sigma_{i-1}^{y} \sigma_{i+1}^{y}\right] \\
& +\frac{1}{4} \sum_{i}\left(1+\alpha_{K} \sigma_{i}^{z} \sigma_{i+1}^{z}\right)+\frac{1}{2} \epsilon_{-K} \sum_{i} \sigma_{i}^{z}, \quad K>0
\end{aligned}
$$

As can be readily verified from this equation, in the limit $T$ $\rightarrow 0^{+}$the action of $H_{\text {kink }}$ on any configuration having non NN kinks vanishes like $O\left(e^{-4 K}\right)$, thus yielding a metastable state. In the spin representation this corresponds to configurations with domains lengths larger than the lattice spacing, which amounts to hindering spins to detach and so diffuse through. In turn, from Eq. (10) it can be checked that this situation also yields metastable states of $H_{\text {spin }}$ so long as $J>0$. Clearly, the number of these configurations grows exponentially with the system size, and most of them take over the asymptotic dynamics with long lifetimes $\propto \xi^{2}$. This marks an important difference with respect to the $\mathrm{AF}$ dynamics to be introduced briefly in Sec. II B.

As an aside, we finally mention that by construction $H_{\text {kink }}$ not only preserves the parity of kinks $e^{i \pi \Sigma_{j} \hat{n}_{j}}$ (being even for PBC) but in turn satisfies

$$
\left[H_{\text {kink }}, \sum_{j} \sigma_{j}^{z} \exp \left(i \pi \sum_{k \leq j} \hat{n}_{k}\right)\right]=0
$$

which simply expresses the conservation of the total spin magnetization in the original system. In practice, for the numerical evaluation of gaps we will just build up the adequate kink states from the corresponding spin ones.

\section{B. Antikinks: $\boldsymbol{J}<\mathbf{0}$}

While most of the above ideas and procedures applies to the AF dynamics as well, ultimately the relaxation to equilibrium becomes faster than in the $\mathrm{F}$ case. By changing from a description based on kinks to AF domain walls, i.e., to antikinks, we can easily construct an evolution operator that for large times and LT regimes essentially describes a diluted antikink system. As before, if we think of antikinks as hard core particles $A$ (former vacancies $\varnothing$ under F couplings), the basic processes now involve (i) next NN hopping, i.e., $A$ $+\varnothing+\varnothing \rightleftharpoons \varnothing+\varnothing+A$, and (ii) deposition-evaporation "assisted" by vacancies, that is, $\varnothing+\varnothing+\varnothing \rightleftharpoons A+\varnothing+A$.

With the aid of these schematic events, illustrated, respectively, in Figs. 3(a) and 3(b), and after carrying out the rotation referred to in Eq. (14) but with an argument $\bar{\varphi}=-i K$, the symmetric representation of the antikink operator can be finally cast as

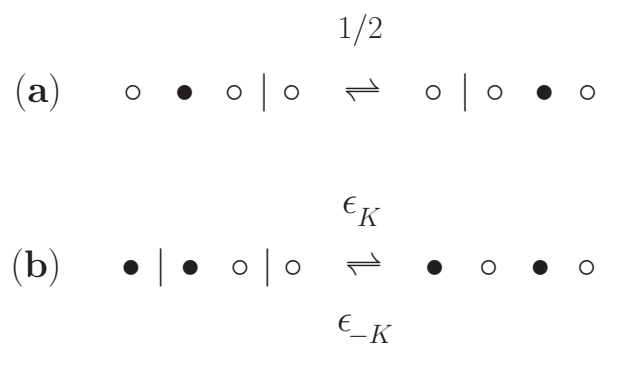

FIG. 3. Transition rates for (a) next-nearest-neighbor antikink hoppings mediated by vacancies and (b) assisted depositionevaporation of antikinks. As in Fig. 2, these latter are represented by vertical lines but separating antiferro domains.

$$
\begin{aligned}
H_{\mathrm{anti}}= & \frac{1}{8} \sum_{i}\left(1-\sigma_{i}^{z}\right)\left[\left(1+\gamma_{K}\right) \sigma_{i-1}^{x} \sigma_{i+1}^{x}+\left(1-\gamma_{K}\right) \sigma_{i-1}^{y} \sigma_{i+1}^{y}\right] \\
& +\frac{1}{4} \sum_{i}\left(1+\alpha_{K} \sigma_{i}^{z} \sigma_{i+1}^{z}\right)-\frac{1}{2} \epsilon_{-K} \sum_{i} \sigma_{i}^{z}, \quad K<0 .
\end{aligned}
$$

Of course, by exchanging the role of particles and vacancies, i.e., using the canonical transformation $\sigma^{ \pm} \rightarrow \sigma^{\overline{+}}$, one recovers the form of Eq. (18), but this likelihood is only superficial. Note that for $K \rightarrow-\infty$ the effect of the uniform field $\epsilon_{-K}$ vanishes like $O\left(e^{4} K\right)$, so the only jammed state on which the action of the diagonal terms cancels out at this order is the vacuum of antikinks. Thus, in the limit $T \rightarrow 0^{+}$the dynamics is no longer dominated by metastable states (instead proliferating exponentially for $J>0$ ). In this situation AF domains can grow unhindered, as single antikinks now detach and diffuse freely (Fig. 3), thus allowing the dynamics to run smoothly toward equilibrium. As a result the dynamic exponent, whose numerical analysis we next turn to consider, becomes smaller (actually diffusive) than for $\mathrm{F}$ exchanges.

\section{NUMERICAL RESULTS}

We now investigate numerically the spectral gaps of the Kawasaki operators given in Eqs. (10), (18), and (20) along with their dynamic exponents in different situations. As a consistency check first we verified that the (rotated) Boltzmann distribution corresponds in fact to the steady state of those operators. This also served to start up the Lanczos recursion with a random state but chosen orthogonal to that equilibrium configuration. Thereafter we obtained the first excited eigenmodes of our symmetric $H$ s using periodic chains of up to 24 sites, the main limitation for this being the exponential growth of the space dimensionalities. Another restrictive issue is that below $k_{B} T /|J| \sim 0.1-0.2$ the Lanczos convergence slows down progressively because in most situations the spacing of low lying levels turns out to decay as $e^{-4|K|}$. Thus, in what follows we content ourselves with giving results above that region where nonetheless clear saturated tendencies can be already obtained.

\section{A. $\boldsymbol{J}>\mathbf{0}$}

Turning to the F dynamics, in the insets of Figs. 4(a) and 4(b) we exhibit the finite-size behavior of spectral gaps for 

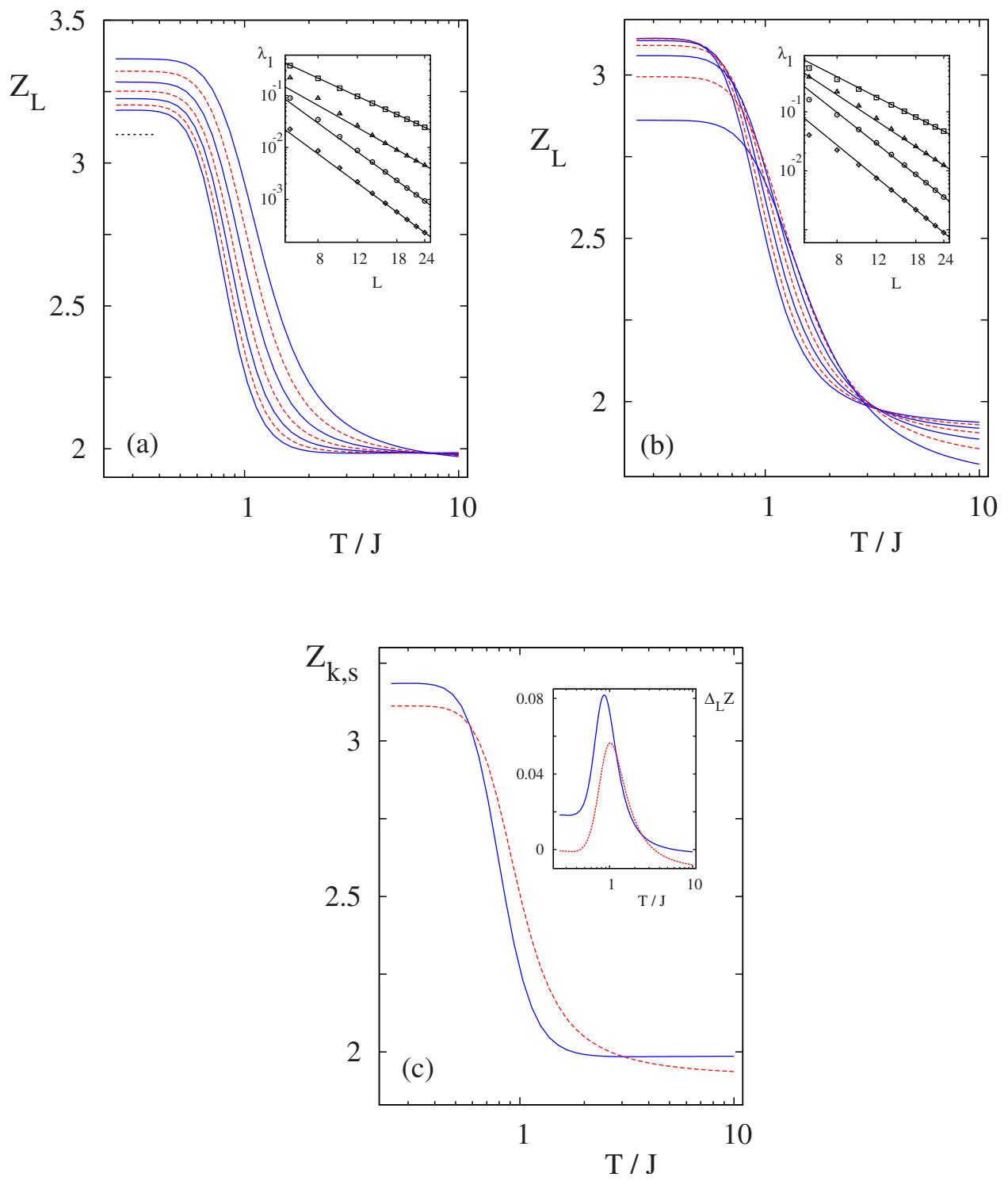

FIG. 4. (Color online) Effective dynamic exponents of Eq. (21) for $J>0$ using $12 \leq L \leq 24$ with $L$ even, computed for (a) spins and (b) kinks. Sizes increase downward almost throughout (a) and upward in (b) at both leftmost and rightmost temperature regions. The respective insets display the gap behavior with the system size for $T / J=10,1,1 / 2$, and $1 / 4$ (top to bottom). To compare slopes, data have been shifted upward with respect to $T / J=10$. Straight lines are fitted with slopes $-z_{24}(T)$ as calculated from the main panels. In (c) we compare these exponents for kinks and spins (dashed and solid lines). At low as well as at high temperatures $z_{k}$ and $z_{s}$ yield, respectively, lower and upper bounds for the thermodynamic limit of $z$. The inset of (c) provides a measure of convergence for these quantities by depicting $\Delta_{L} z \equiv z_{L-2}$ $-z_{L}$ for our maximum sizes. Horizontal short doted line in (a) stands for the extrapolations referred to in the text.

the case of spins and kinks, respectively. They are all consistent with a gap vanishing like $1 / L^{z}$ although with a nonuniversal temperature dependent dynamic exponent $z(T)$. For the purpose of observing in more detail the trend of size effects on these exponents under different temperature regimes, we considered a sequence of effective approximants defined as

$$
z_{L}=\frac{\ln \left[\lambda_{1}(L-2) / \lambda_{1}(L)\right]}{\ln [L /(L-2)]}
$$

( $L$ even), which simply provide successive measures of the gap closing in either of the above representations. These are shown in the main panels of Figs. 4(a) and 4(b). As expected, in the HT region $H_{\text {spin }}$ is able to yield fairly convergent estimations of $z(\sim 1.99)$, so recapturing the plain diffusive limit above $T / J \simeq 3$ (henceforth the Boltzmann constant $k_{B}$ is set equal to 1). Besides, in this representation the above approximants turn out to constitute upper bounds of this exponent for almost all temperatures studied, though exhibiting different spreadings. We attempted to extrapolate these bounds using several procedures, but owing to the variable spreading this resulted in a rather noisy limiting curve. Nevertheless, in the region that most interests us the spreading becomes more stable, thus upon using a van den Broeck-Schwartz extrapolation scheme [15] we found that $z \sim 3.10$, in reasonable agreement with analysis and Monte Carlo simulations under instantaneous quenches [7]. Moreover, this result is also con- 


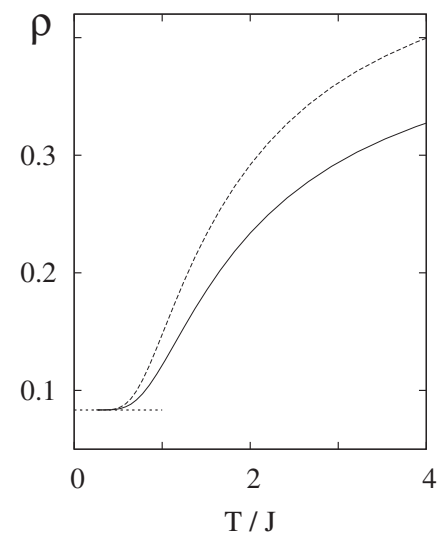

FIG. 5. Density of kinks for the first excitation (solid line) and ground state (dashed line) of $\mathrm{H}_{\text {kink }}$ [Eq. (18)] with $L=24$ and $J$ $>0$. Lowermost dots indicate the equilibrium density $(2 / L)$ when $T \rightarrow 0$.

sistent with estimations of $z$ arising from $H_{\text {kink }}$ approximants around the same region $(T / J \lesssim 0.4)$ where, as discussed above, the kink description in finite sizes is most reliable. In fact, no extrapolations are needed here since already the values of $z_{20} \lesssim z_{22} \lesssim z_{24}$ follow very closely one another, collapsing near a value of $z \simeq 3.11$. This is the main result of this section.

As an added bonus, note that in the LT regime the kink approximants come out conforming a sequence of lower bounds which conveniently complement the upper ones provided by the spin representation. This is illustrated in Fig. 4(c) where for $T / J \lesssim 0.4$ both kink and spin approximants should actually enclose the value of $z$ in the limit $L \rightarrow \infty$ (presumably much closer to the kink border). Using the results of our maximum reachable sizes, this suggests that in the ordering regime the dynamic exponent is bounded as $3.11 \leqq z<3.18$. For $0.5 \leqq T / J \leqq 2$ the trend of finite sizes of the kink approximants now reverses and, alike the spin $z_{L} \mathrm{~S}$, turn out to yield upper bounds of $z$. The kink approximants converge slightly faster in this region [see inset of Fig. 4(c)], until for $T / J \gtrsim 2$ the trend of bounds reverses once more. Hence, at HT scales the actual value of $z$ is enclosed again by our kink and spin approximants though this time these latter take the lead, as it should.

As mentioned earlier, the LT convergence of kink approximants was somehow foreseeable on the basis of equilibrium considerations but it is not yet clear whether these apply to nonequilibrium as well. In Fig. 5 however we show that this also the case, at least for the sizes at hand. There, we display the kink density $\rho_{1}=\frac{1}{L} \sum_{i}\left\langle\psi_{1}\left|\hat{n}_{i}\right| \psi_{1}\right\rangle$ for the first excited mode of $H_{\text {kink }}$ which for $T / J \leqq 1$ closely follows the diluted kink picture already expected for equilibrium, i.e., $\rho_{1} \rightarrow 2 / L$. Also, preliminary evaluations of kink-kink correlations $\left\langle\psi_{1}\left|\hat{n}_{i} \hat{n}_{j}\right| \psi_{1}\right\rangle$ show that these become negligible below $T / J \sim 0.5$, thus resembling the fully segregated equilibrium state. In passing it is worth pointing out that while $\lambda_{1}$ is doubly degenerate, the correlations and $\rho_{1}$ remain indistinguishable in both of these eigenmodes.

$$
\text { B. } \boldsymbol{J}<\mathbf{0}
$$

In studying numerically the AF dynamics, the approximants Eq. (21) pose severe difficulties at intermediate tem-
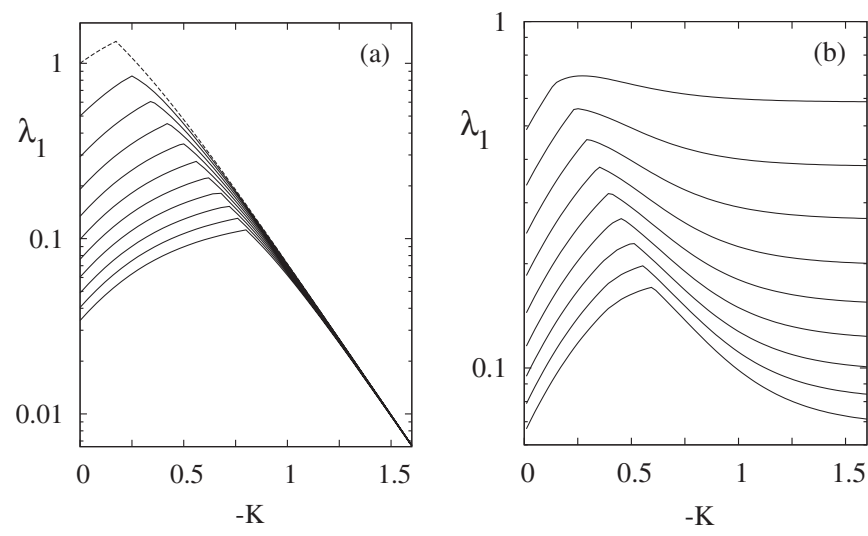

FIG. 6. Spectrum gaps of (a) spin and (b) antikink operators [Eqs. (10) and (20), respectively] for various inverse temperatures $K=J / T,(J<0)$. Sizes increase from top to bottom. In (a) the dashed line stands for the soluble case $L=4$ already indicating the appearance of a cusp due to level crossings [see Eq. (22)], a feature holding also for $L=6,8, \ldots, 24$. At low temperatures $\lambda_{1}(L)$ decays as $e^{-4|K|}$ with an almost size independent amplitude. In (b) this latter problem is remedied although level crossings for $8,10, \ldots$, and 24 sites now give rise to nonanalyticities near which size effects become severe (cf. Fig. 7).

peratures. Owing to low lying level crossings, now the spectrum gap is bisected in temperature regions having opposite monotonic behaviors. For instance, it is instructive to consider the case of $L=4$ where this feature already appears in the spectrum of $H_{\text {spin }}$. By diagonalizing its associated $6 \times 6$ stochastic matrix, we find that there are two branches of eigenvalues crossing at $K^{*}=-\frac{1}{4} \ln 2$, thus producing the nonanalytic gap

$$
\lambda_{1}(4)= \begin{cases}2 \epsilon_{K}, & \text { threefold degenerate if } K \geq K^{*}, \\ 4 \epsilon_{-K}, & \text { nondegenerate otherwise }\end{cases}
$$

as seen in the uppermost curve of Fig. 6(a). Alike this simple case, for larger sizes it is found numerically that $\lambda_{1}(L)$ remains degenerate only above certain temperatures below which level crossings occur, and $\lambda_{1}$ results in a nondegenerate value. This is signaled by the emergence of the cusps observed in Fig. 6(a). At LT regions the gap recovers the exponential decay referred to above but the data collapse now precludes finite-size estimations of effective exponents from Eq. (21). Although level crossings come out at successively smaller temperatures, these cannot keep pace with the increasing lattice sizes. To bypass these limitations we resorted to the antikink operator especially constructed for this region [Eq. (20)]. As can be seen in Fig. 6(b), now the LT spectrum no longer collapses so the approximants Eq. (21) can be employed once more. However, as evidenced by the cusps of that figure, the problem of low lying level crossings yet persists. In nearing the crossing temperatures this brings about pronounced size effects in $z_{L}$, partly because of the mismatches occurring between eigenvalue branches, alike those observed in Fig. 6(a). 


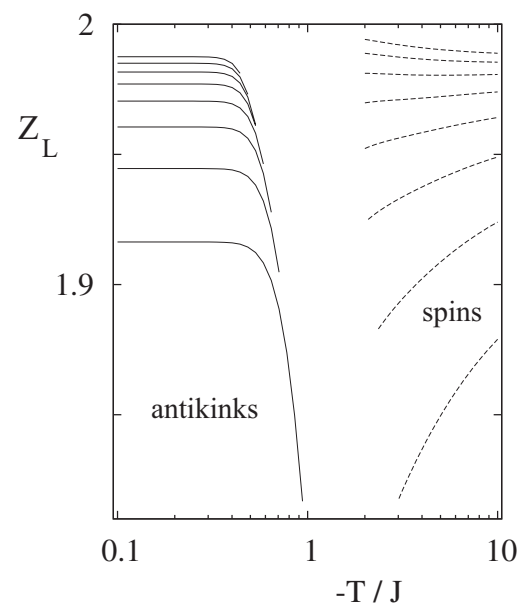

FIG. 7. Effective dynamic exponents as defined in Eq. (21), for Hamiltonians (10) and (20) with $J<0$ and $L=10,12, \ldots, 24$ increasing upward. In nearing the cusps of Fig. 6 size effects become progressively pronounced, i.e., $\lambda_{1}(L)$ and $\lambda_{1}(L-2)$ belong to different branches. High- and low-temperature regimes are both consistent with a typical diffusive exponent $\left(z_{24} \simeq 1.99\right)$.

All the above considerations result in fair convergent estimations of dynamic exponents both for LT and HT regimes, but as displayed in Fig. 7, the intermediate zone is out of reach. Despite this setback, what matters is the usefulness of the antikink representation Eq. (20) in the more interesting LT region which otherwise would have remained inaccessible. As in the F case, no extrapolations to the thermodynamic limit are needed here since already for $T /|J| \lesssim 0.3$ our higher antikink approximants lie within the interval 1.98 $\simeq z_{20} \lesssim z_{22} \lesssim z_{24} \simeq 1.99$, thus indicating a Glauber universality class for this ordering regime, cf. [10].

In the other extreme, when disorder prevails, already for $T /|J| \geqslant 3$ the spin approximants derived from the data of Fig. 6(a) converge within one percent to our LT exponents, thus suggesting for these latter a rather robust diffusive behavior.

Finally, in Fig. 8 we exhibit the antikink density $\rho_{1}$ for the first excited state of $H_{\text {anti. }}$. As before, this confirms the diluted antikink picture expected at LT scales, in turn accounting for the convergent approximants obtained below $T / J \leqq 0.3$. However, this time $\rho_{1}$ does not quite follow there the vanishing equilibrium density of the plain $\mathrm{AF}$ vacuum state and adopts instead the limiting value referred to in Fig. 5, indicating the presence of two antikink excitations. At intermediate temperatures the jump of $\rho_{1}$ just reflects the aforementioned level crossings.

\section{CONCLUDING DISCUSSION}

To summarize, we have constructed symmetric representations of the Kawasaki dynamics in the Ising chain using a quantum spin analogy with both direct and dual processes. The kink description [7], either in its F or AF versions [Eqs. (18) and (20)], is well suited to low-temperature regimes where it is able to provide a rather fast finite-size convergence to dynamic exponents (Figs. 4(b) and 7). For F couplings, the ordering kinetics arising from these latter $(z$

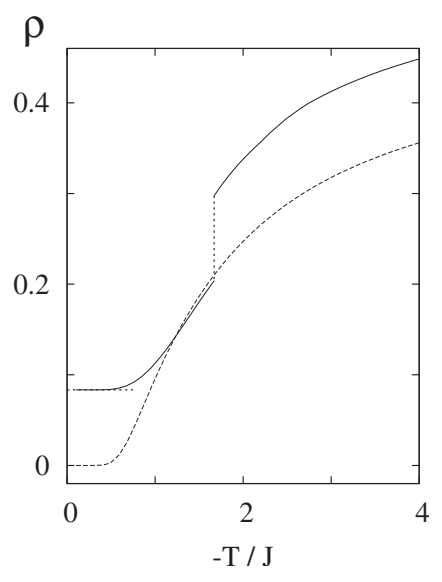

FIG. 8. Density of antikinks for the first level and ground state of operator Eq. (20) with $L=24$ and $J<0$ (solid and dashed lines respectively). For comparison, horizontal dots indicate the equilibrium density of ferro kinks $(J>0)$ at $T=0$, cf. Fig. 5. The discontinuity denoted by vertical dots stems from level crossings in the low lying spectrum of $H_{\text {anti }}$ [also, see cusp in the lowermost curve of Fig. 6(b)].

23.11) turns out to be slightly slower than the LifshitzSlyozov behavior $\left[\xi(t) \propto t^{1 / 3}\right]$ characteristic of higher dimensions [9]. By contrast, for $\mathrm{AF}$ interactions the kinetics is no longer activated by metastable states, and dynamic exponents converge rapidly $(z \simeq 1.99)$ to the typical diffusive values of the Glauber universality class $\left[\xi(t) \propto t^{1 / 2}\right]$.

On the other hand, the spin representation [Eq. (10)] is more appropriate for higher temperature scales where the kinetics remains slow and is still characterized by relaxation times diverging as $L^{z}$ [inset of Fig. 4(a)]. For the F dynamics, the finite-size approximants of these exponents turn out to form a sequence of upper bounds which also extends down to low-temperature regions [Fig. 4(a)], where it nicely complements the series of lower bounds emerging from the kink approach [Fig. 4(b)]. On wider temperature scales, both representations are consistent with a nonuniversal set of dynamic exponents interpolating continuously between a subdiffusive ordering kinetics and the plain diffusive limit [Fig. 4(c)]. In this regard, the field theory associated to Eqs. (10) and (18) becomes rather involved due to the correlated hopping terms appearing in those pseudo-Hamiltonians. Hence, the eventual emergence of marginal operators accounting for such nonuniversality is an open issue which deserves further investigation. For $J<0$, part of the intermediate temperature region proved inaccessible due to size effects caused by low lying level crossings in the spectrum of both spin and antikink operators (cusps of Fig. 6). However, as the dynamics of this case is not activated, the diffusive exponents obtained by both representations within their natural range of applicability (Fig. 7) suggest a rather universal (Glauber) dynamics throughout.

The success of the kink description at low-temperature regimes would probably allow to extend the ideas of this work to other interesting 1D-dynamics under instantaneous quenches (i.e., constant transition rates). For instance, the nonuniversal behavior alleged for the ordering kinetics of the alternating bond Kawasaki chain [10], might well be further 
investigated with our numerical approach. Other lines of research that would also be worth pursuing are certain dynamical processes involving composite objects (e.g., dimers) which exhibit strongly broken ergodicity as a result of having an extensive number of conservation laws [16]. As the issue of universality classes in nonequilibrium statistical systems is often linked to the existence of these latter [17], it would be important to determine whether dynamic exponents actually depend on the subspaces where the evolution takes place. Dual representations constructed for such processes could therefore provide reliable computations of these expo- nents within ordering regimes. Further work in this direction is in progress.

\section{ACKNOWLEDGMENTS}

The author wishes to thank M. Arlego, G. L. Rossini, and F. A. Schaposnik for helpful comments and discussions, as well as D. Dhar, S. Redner, and G. Ódor for helpful observations and correspondence. Support of CONICET, Argentina under Grants No. PIP 1691 and No. PICT ANCYPT 20350 is acknowledged.
[1] For reviews consult, for instance, A. J. Bray, Adv. Phys. 43, 357 (1994); J. D. Gunton, M. San Miguel, and P. S. Sahni, in Phase Transitions and Critical Phenomena, edited by C. Domb and J. L. Lebowitz (Academic Press, London, 1983), Vol. 8; K. Kawasaki, in Phase Transitions and Critical Phenomena, edited by C. Domb and M. S. Green (Academic Press, London, 1972), Vol. 2.

[2] R. J. Glauber, J. Math. Phys. 4, 294 (1963); B. U. Felderhof, Rep. Math. Phys. 1, 215 (1971).

[3] K. Kawasaki, Phys. Rev. 145, 224 (1966).

[4] At a coarse grained or hydrodynamic level of description both dynamics pertain to the classification scheme of P. C. Hohenberg and B. Halperin, Rev. Mod. Phys. 49, 435 (1977).

[5] Nonequilibrium Statistical Mechanics in One Dimension, edited by V. Privman (Cambridge University Press, 1997).

[6] More recently, tagged diffusion and metastable aspects of the 1D-Kawasaki dynamics have been investigated by C. Godrèche and J. M. Luck, J. Phys. A 36, 9973 (2003); G. De Smedt, C. Godrèche, and J. M. Luck, Eur. Phys. J. B 32, 215 (2003).

[7] S. J. Cornell, K. Kaski, and R. B. Stinchcombe, Phys. Rev. B 44, 12263 (1991); see also S. J. Cornell in Ref. [5] and references therein.

[8] A. J. Bray, Phys. Rev. Lett. 62, 2841 (1989) consult also A. Bray in Ref. [5].
[9] D. A. Huse, Phys. Rev. B 34, 7845 (1986); I. M. Lifshitz and V. V. Slyozov, J. Chem. Solids 19, 35 (1961).

[10] J. H. Luscombe, Phys. Rev. B 36, 501 (1987); J. M. Nunes da Silva and E. J. S. Lage, Phys. Rev. A 40, 4682 (1989).

[11] It has been argued that such value might be an upper bound to the dynamic exponent, see R. Cordery, S. Sarker, and J. Tobochnik, Phys. Rev. B 24, 5402 (1981); Reference [7] indicates that $z=5$ actually corresponds to a slow cooling regime.

[12] W. Zwerger, Phys. Lett. A 84, 269 (1981).

[13] See, for example, G. H. Golub and C. F. van Loan, Matrix Computations, 3rd ed. (Johns Hopkins University Press, Baltimore, 1996); G. Meurant, The Lanczos and Conjugate Gradient Algorithms (SIAM, Philadelphia, 2006).

[14] N. G. van Kampen, Stochastic Processes in Physics and Chemistry, 3rd ed. (North Holland, Amsterdam, 2007).

[15] J.-M. Vanden Broeck and L. W. Schwartz, SIAM J. Math. Anal. 10, 658 (1979); C. J. Hamer and M. N. Barber, J. Phys. A 14, 2009 (1981).

[16] G. I. Menon, M. Barma, and D. Dhar, J. Stat. Phys. 86, 1237 (1997);

M. Barma, M. D. Grynberg, and R. B. Stinchcombe, J. Phys.: Condens. Matter 19, 065112 (2007).

[17] G. Ódor, Rev. Mod. Phys. 76, 663 (2004). 